\title{
Electrochemical detection of TNT at cobalt phthalocyanine mediated screen-printed electrodes and application to detection of airborne vapours.
}

J. Sarah Caygill ${ }^{1}$, Stuart D. Collyer ${ }^{2}$, Joanne L. Holmes ${ }^{1}$, Frank Davis ${ }^{1}$, Séamus P.J. Higson ${ }^{1}$ *

${ }^{1}$ Cranfield Health, Vincent Building, Cranfield University, Bedfordshire, MK43 OAL, UK.

${ }^{2}$ Microarray Ltd, Cranfield Biotechnology Centre, Cranfield University, Cranfield, MK43

$O A L, U K$.

* Corresponding author. Tel: $+44(0)-1234-758516$. Fax: $+44(0)-1234-758380$. Email address: s.p.j.higson@cranfield.ac.uk

\begin{abstract}
We describe the use of cobalt phthalocyanine as a mediator to improve the sensitivity for the electrochemical detection of TNT. Commercial screen-printed electrodes containing cobalt phthalocyanine were employed for determination of TNT. Improved sensitivities compared to screen-printed carbon electrodes without phthalocyanine were observed, current response for cyclic voltammetric measurements at modified electrodes being at least double that of unmodified electrodes. A synergistic effect between oxygen and TNT reduction was also observed. Correlation between TNT concentrations and sensor output was observed between 0-200 $\mu \mathrm{M}$ TNT. Initial proof-of-concept experiments combining electrochemical determinations, with the use of an air-sampling cyclone, are also reported.
\end{abstract}

Keywords: TNT; cyclic voltammetry; air sampling; cyclone; electrochemistry 


\section{Introduction}

Recent demands for improved homeland security, along with the need to detect hidden ordnance, such as roadside bombs or Improvised Explosive Devices (IEDs), has been the driving force behind increased research efforts into the detection of trace explosive materials. The current state-of-the-art technologies are based on mass spectrometry and are capable of detecting airborne explosive vapours at low levels, however, these systems can be both bulky and expensive. Other methods include the swabbing of suspects hands and examining these samples using techniques such as ELISA assays, however the time required to carry out the assay (usually requiring sending samples to a dedicated laboratory) renders this technique unsuitable for giving an "instant" answer. For in-field detection of explosives such as trinitrotoluene (TNT) a rapid, sensitive and portable detection system is required [1,2].

Many commercially available sensors are electrochemical in nature, due to the inherent advantages of relatively low cost production, enhanced sensitivity and relatively small equipment size required for these systems. Electrochemical methods are especially suitable for detecting TNT, and related dinitrotoluene (DNT), since both compounds readily undergo a number of redox transformations [3]. Screen-printed electrodes have been previously utilised for electrochemical determination of aqueous TNT with detection limits of $200 \mu \mathrm{g} \mathrm{L}^{-1}$ [4]. Detection limits of $30 \mu \mathrm{g} \mathrm{L} \mathrm{L}^{-1}$ were reported by use of carbon fibre electrodes [5]. Screenprinted carbon electrodes have been modified with polymers to adsorb and concentrate TNT, with typical materials including agarose [6] and Nafion [7], which allowed quantification of levels of TNT as low as $30 \mu \mathrm{g} \mathrm{L}^{-1}$ in spiked lake water. Wearable detectors for TNT have been constructed by depositing screen-printed electrodes onto Gore-Tex [8]. Other electrochemical systems for TNT include the use of mercury-gold amalgam electrodes [9], lowering detection limits to $7 \mu \mathrm{g} \mathrm{L}^{-1}$. Carbon nanomaterials have also been incorporated into these systems, and carbon nanotube containing electrodes have been utilised to reduce detection limits to $7 \mu \mathrm{g} \mathrm{L}^{-1}[10]$. Similar detection levels for TNT in environmental water samples have been achieved using graphene modified electrodes [11,12]. Use of many of these nanostructured materials however could add to the cost of resultant devices, and therefore in earlier work we reported the use of inexpensive, mass-produced commercial screen-printed carbon electrodes for the electrochemical determination of TNT and the related explosive DNT, with linear ranges from 1-200 $\mu \mathrm{M}$ and limits of detection for TNT and DNT as low as $0.4 \mu \mathrm{M}\left(90 \mu \mathrm{g} \mathrm{L}^{-1}\right)$ and $0.7 \mu \mathrm{M}\left(130 \mu \mathrm{g} \mathrm{L}^{-1}\right)$ respectively [13]. 
Within this work we continue and expand our previous work on the electrochemical detection of TNT [13]. Previous work demonstrated that aqueous TNT could be detected at screen printed carbon electrodes [13]. We report several advances here, one of which being that the use of CoPc modified electrodes greatly increases the signal response compared to similar electrodes that do not contain the mediator.

One major drawback for electrochemical detection systems is that they normally can only be applied to aqueous samples, although this could still be useful, for example, for the detection of buried or sequestered explosives due to ground water contamination, or in assessing the presence of TNT in contaminated soils. Since detection of TNT vapour is much more likely to be of application in determining the presence of explosive devices, we also attempted to analyse airborne TNT. Therefore within this work we have developed a proof of principle that a suitable air sampling system can be used to extract TNT from air and concentrate it down into an aqueous solution that can then be interrogated electrochemically. A commercially available cyclone collection system, the Coriolis ${ }^{\circledR} \mu$ air sampler (Bertin Technologies), which is a condensing system that draws in large quantities of air at speeds of up to 300 litres per minute was utilised in a proof-of-concept study. This enables the collection of airborne pollutants, present in low levels, to be concentrated within a small volume of liquid. Air is aspirated into the collection cone, which has been primed with an aliquot of collection fluid, in a whirling motion that forms a vortex. This pulls the airborne particles against the walls due to the centrifugal force exerted which enables collection in the form of a concentrated fluid. This liquid can then be analysed for any airborne contaminants present. Other workers have already utilised a commercial cyclone to extract and concentrate airborne TNT into a water sample, the resultant sample being assayed using a monoclonal antibody to TNT combined with a fluorescence detection method [14].

\section{Materials and methods}

\section{Reagents and materials}

$\mathrm{NaH}_{2} \mathrm{PO}_{4}, \mathrm{Na}_{2} \mathrm{HPO}_{4}$ and $\mathrm{NaCl}$ (all AnalaR grade) were obtained from BDH Laboratory Supplies (Poole, Dorset, UK). 2,4,6-Trinitrotoluene (1000 $\mu \mathrm{g} \mathrm{ml}^{-1}$ in acetonitrile) was obtained from Supelco (Bellefonte, Pennsylvania, USA). Acetonitrile (AnalaR), Sulphuric acid (98\%) and hydrogen peroxide (30\%) were purchased from Fisher Scientific 
(Loughborough, Leicester, UK). Ferrocene carboxylic acid (FCA) was purchased from Sigma-Aldrich.

All solutions were prepared with deionised water ( $\geq 18 \mathrm{M} \Omega \mathrm{cm}^{-1}$ resistivity) from an Elga Purelab UHQ-II Water System (Vivendi Water Systems, High Wycombe, Buckinghamshire, UK). A pH 7.0 phosphate buffer solution (PBS) was prepared, comprising: $4.3 \times 10^{-3} \mathrm{M}$ $\mathrm{NaH}_{2} \mathrm{PO}_{4}, 5.6 \times 10^{-3} \mathrm{M} \mathrm{Na}_{2} \mathrm{HPO}_{4}$ and $7.87 \times 10^{-2} \mathrm{M} \mathrm{NaCl}$.

A Piranha solution consisting of a $3: 1$ combination of $98 \%$ sulphuric acid and $30 \%$ hydrogen peroxide was used for the removal of organic residues from the glass cell used in electrochemical interrogations between experimental procedures (caution - Piranha is highly oxidising and corrosive).

The commercial screen printed carbon electrodes (SPCE) were obtained from Microarray (Manchester, UK) and comprised of a 4-electrode system with two carbon ink working electrodes (electroactive area of $22 \mathrm{~mm}^{2}$ per WE), a carbon ink counter electrode and a silver/silver chloride reference electrode. Cobalt phthalocyanine (CoPc) electrodes were identical apart from the fact that the working electrodes had been fabricated using a carbon ink doped with 5\% CoPc (w/w). Scanning Electron Microscopy (SEM) images were obtained using a Philips XL30 SEM. Samples were sputter coated with gold-palladium before imaging.

\section{Electrochemical measurements}

All electrochemical studies were made using an analytical electrochemical workstation (PG580) from Uniscan Instruments (Buxton, UK) which utilised inherent software supplied with it for recording the current/charge outputs resulting from sensor interrogations. A sealed prototype electrochemical cell was designed in-house and fabricated by Soham Scientific (Ely, Cambridge, UK), as described in previous work [13].

Cyclic voltammetry (CV) measurements were performed on a single working electrode of the screen-printed carbon (or CoPc) sensors in the sealed glass cell described above. The TNT solutions were prepared in the $\mathrm{pH}$ 7.0 PBS. When required, the cell was deoxygenated by gentle purging with argon (BOC, Letchworth, UK). Cyclic voltammetric investigation of TNT was performed by sweeping the potential between -0.8 and $+0.8 \mathrm{~V}$ (vs. $\mathrm{Ag} / \mathrm{AgCl}$ ) for one cycle; the scan rate for all interrogations was $20 \mathrm{mVs}^{-1}$. 
An air sampling system was used for all collection studies of airborne particles, the Coriolis ${ }^{\circledR} \mu$ which was loaned by Leeds University. The Coriolis ${ }^{\circledR} \mu$ air sampling device was set to run for 2 minutes at the maximum collection setting, 300 litres per minute. A $10 \mathrm{ml}$ solution of $\mathrm{pH}$ 7.0 PBS was used as the collection fluid - this volume was specified in the instructions as required to enable the development of a vortex when the device was in operation. A spray bottle was used to aspirate the sample (FCA or TNT) in the vicinity of the air inlet valve. One aspiration was performed every 10 seconds for the entire 2 minutes. The resulting sample, collected in the collection fluid, was interrogated electrochemically as previously described with a CoPc electrode. Weighing the spray bottle before and afterwards gave the amount of solution expelled.

Any observed increases in the electrochemical activity gained when utilising cobalt phthalocyanine doped screen-printed electrodes, when compared to the response obtained with plain carbon screen printed electrodes (i.e. undoped), is postulated to be due to the inherent mediating activity of the CoPc species. Cobalt phthalocyanine, can act as an electrocatalyst when adsorbed onto carbon electrodes [15] enabling reactions such as oxygen reduction, carbon dioxide reduction and the oxidation of thiols to be catalysed by the presence of the metallophthalocyanine complex.

\section{Results and Discussion}

\section{Characterisation of electrodes}

An examination of the surface topography of the cobalt phthalocyanine screen-printed carbon electrode revealed the presence of particles of the CoPc clearly within the electrode matrix (figure 1b). When comparing the surface to the plain carbon sensors (figure 1a) there are clear differences in the $\mathrm{CoPc}$ sensor image, with areas of greater conductivity, as demonstrated by the greater luminescence around the edges of these particles compared to the mostly featureless topography of the carbon surface.

\section{Cyclic Voltammetry of TNT}

Our previous investigations have shown that the electrochemical interrogation of TNT with the plain carbon sensor platforms utilised within these studies yields a current response due to the electroactivity of TNT itself [13]. The mechanism by which this occurs (figure 1c), a four electron reduction of each nitro group to hydroxylamine followed by a two electron reduction 
from hydroxylamine to amine, has been described earlier $[3,13]$. The motivation for utilising the cobalt phthalocyanine doped sensors was that an improvement in current response and limits of detection (when compared to un-doped carbon inks) might therefore be demonstrated in the presence of TNT and this would improve the applicability of this technique in the sphere of explosive detection.

Cobalt phthalocyanine ink modified electrodes were chosen since they display catalytic properties towards a variety of electrochemical reactions by acting as mediators [16,17]. Cyclic voltammetry of PBS solution was used as a control to determine any activity of the electrodes before exposure to TNT. A small reductive peak was observed (figure 2) for the CoPc electrodes in pH7.0 PBS whereas no peak was observed for the plain carbon electrodes under the same conditions. It has been previously demonstrated that a reductive process occurring in the macrocyclic $\mathrm{CoPc}$ molecule on screen-printed carbon produced a reductive peak at $-0.30 \mathrm{~V}$ (vs. SCE in PBS) [16]. Other work demonstrated a reductive peak at $-0.60 \mathrm{~V}$ (vs. SCE in PBS) at a CoPc modified graphite electrode [17]. The reductive peak observed in the voltammogram obtained from our system is between those reported in the literature (vs. $\mathrm{Ag} / \mathrm{AgCl})$ and may be attributed to the electrochemical reduction of $\mathrm{Co}(\mathrm{II})$ to $\mathrm{Co}(\mathrm{I})$.

PBS solutions with/without $200 \mu \mathrm{M}$ TNT were interrogated using cyclic voltammetry and the CoPc-doped screen printed electrodes (figure 2). The voltammogram show similarities to those obtained for plain carbon screen printed electrodes (SPCE) in our original study (which have been overlaid here to allow for direct comparison) [13]. There is a redox couple present about the $0.0 \mathrm{~V}$ potential position (at +0.01 and $-0.11 \mathrm{~V}$ vs. $\mathrm{Ag} / \mathrm{AgCl}$ ) which can be related to those previously reported [13] at +0.30 and $-0.30 \mathrm{~V}$, known as $\mathrm{O} 1$ and R2, when TNT is interrogated with a plain carbon SPCE system.

There are also significant reductive peaks present in the cathodic region at -0.50 and $-0.77 \mathrm{~V}$. This aspect differs from the voltammograms gained on plain carbon [13] where there is only one reductive peak present in this region, $\mathrm{R} 1$ at $-0.72 \mathrm{~V}$. It is postulated that the mediating effect from the CoPc doped sensor has not only significantly increased the current response but has also enabled the splitting of the original single peak within this reductive region to a form that better represents the sequential electrochemical reduction of the nitro groups on the TNT molecule into hydroxylamine groups [18]. A comparison of peak positions and responses of plain carbon and CoPc doped screen printed electrodes are provided (table 1). 
At negative potentials, the electron deficient nitrogens of the nitro-groups accept electrons and there is a four electron reduction to hydroxylamine groups [3, 19]. Reduction of aromatic nitro groups can occur at varying potentials depending on a number of factors such as the number and relative positions of the nitro groups on the aromatic ring as well as the presence of other substituents and the solution $\mathrm{pH}$. In the case of TNT [3], there are three sequential reductions and these can be observed in our system (shown as R2, R1b and R1a). On the oxidative sweep a single peak is observed $(\mathrm{O} 1)$ which is thought to be due to the oxidation of hydroxylamine groups to nitrosoamines [19].

As can also be seen the use of the CoPc electrodes leads to an increase in sensitivity compared to SPCE (i.e. undoped). This enhancement was observed across the full range of TNT concentrations employed in this study, and figure 3 shows the CVs of these concentrations of TNT between 0-200 $\mu \mathrm{M}$. As can be seen, increased TNT concentrations led to increased electrochemical responses, when directly compared to the original study on undoped SPCEs [18]. This is most likely due to the cobalt phthalocyanine acting as a mediator and facilitating electron transfer between the electrodes and the target molecules.

\section{Effect of oxygen}

The influence of dissolved oxygen on the electrochemical interrogation of nitro compounds using a plain screen printed carbon sensor was examined previously [13]. Purging the plain carbon SPCE electrochemical system containing TNT with argon caused the main reductive peak present at $-0.72 \mathrm{~V}$ to diminish and the redox couple to increase slightly with increasing deoxygenation periods. To eliminate any oxygen influence on the electrochemistry of the reduction of TNT on the CoPc electrodes, the solution was similarly purged with argon and a $\mathrm{CV}$ of $100 \mu \mathrm{M}$ TNT measured after different purging times (figure 4).

It can be observed that the oxidative and reductive peaks behave in the same manner as previously discussed - with the large reductive peak in the cathodic region, R1a, diminishing in size with each increasing deoxygenation period whilst the redox couple (O1 and R2) can be observed to increase with increasing deoxygenation periods.

The new reductive peak, $\mathrm{R} 1 \mathrm{~b}$, at $-0.5 \mathrm{~V}$ is observed to behave in the same manner as reductive peak R1a. This may be associated with the electrochemical breakdown of the dissolved oxygen in the system which can occur at a similar potential to TNT reduction; when this effect is removed, the peak remaining may be directly related to the 
electrochemical breakdown of TNT. The redox couple displays an increased response with increasing deoxygenation time, as observed previously on plain carbon screen-printed electrodes $[13,19]$. This could be due to a competitive process whereby the oxygen dissolved in the system is acting as a competitive electron accepter, thus diminishing the opportunity for the nitroaromatic compound to be reduced as previously reported [19]. All interrogations with the CoPc sensors from this point onwards will be, unless otherwise stated, presented with a 10 minute deoxygenation period, to prevent any possible effects of competitive oxygen interference.

\section{Calibration curves}

When increasing the concentration of the nitroaromatic compound present in the PBS solution it would be expected that the resulting current response would also increase. Therefore progressively stronger concentrations of TNT were interrogated electrochemically. This can be shown as a calibration plot (figure 5) corresponding to peak current values. Data for this calibration chart was taken from the reductive peaks, R1a and R1b, observed at potentials of $-0.72 \mathrm{~V}$ and $-0.50 \mathrm{~V}$ (vs. $\mathrm{Ag} / \mathrm{AgCl}$ ). As outlined previously, these peaks are indicative of the reduction of the nitro groups on the aromatic ring to form hydroxylamine moieties. There were two other peaks present in the voltammograms - one oxidative peak, $\mathrm{O} 1$ at $+0.01 \mathrm{~V}$ and one reductive, $\mathrm{R} 2$, at $-0.11 \mathrm{~V}$ (vs. $\mathrm{Ag} / \mathrm{AgCl}$ ). The anodic peak has been suggested to be the result of oxidation of the hydroxylamine moieties to form nitrosamine groups [19]. The reductive peak present at $-0.11 \mathrm{~V}$ could then correspond to the reduction of these nitrosamine groups back to the hydroxylamine form [19].

Calibration data representing all these reactions can be seen in figure 5. The oxidative peak (O1) provides a quasi-linear calibration plot that has an $\mathrm{R}^{2}$ value of 0.999 up to the $100 \mu \mathrm{M}$ solution concentration position before the influence of the concentration of TNT to the current response begins to level off (Fig 5a). The error bars are included in the calibration profile and represent one standard deviation, calculated from the average of the population, either side of the data point. The peak height data from the reductive peak at $-0.11 \mathrm{~V}$ (vs. $\mathrm{Ag} / \mathrm{AgCl}$ ) provides a linear calibration profile, demonstrating that the electrochemical reduction of TNT in this manner is a reproducible process.

It can be observed that the lower concentrations of TNT interrogated with a CoPc sensor (Fig $5 b$ ) exhibit linear responses with good $\mathrm{R}^{2}$ values at the redox couple of $\mathrm{O} 1$ and $\mathrm{R} 2$, with the oxidation peak in particular demonstrating exceptional linearity with increasing 
concentrations of TNT, $\mathrm{R}^{2}=0.9996$. The error observed when interrogating at this peak is also low, with relatively small error bars. The reductive peak R2 also displays reasonable linearity (both for high and low concentration ranges, Figs $5 \mathrm{a} \& 5 \mathrm{~b}$ respectively). It would appear from these calibration plots that both of these peaks would be suitable for detection of TNT at lower concentrations, with the $\mathrm{O} 1$ peak demonstrating particular suitability due to the high linearity and low error. Limits of detection were obtained by interrogating TNT-free PBS as a zero value and using three times the standard deviation of this value $(n=10)$. The detection limits for these peaks was found to be $1 \mu \mathrm{M}$ for the oxidative peak $\mathrm{O} 1$ and $0.3 \mu \mathrm{M}$ for the reductive peak R2. This is an improvement on that gained with the plain carbon sensors, where the detection limits were found to be 2.6 and $5 \mu \mathrm{M}$ for peak R2 and $\mathrm{O} 1$, respectively [13].

The calibration plots for the peaks R1a and R1b also demonstrate good linearity - this is most apparent with the larger reductive peak, R1a, at the potential position of $-0.77 \mathrm{~V}$. The error in these two reductive peaks has also been demonstrated to be limited as can be observed with the minimal error bars present. The peak R1a has consistently low error in the system which may lend this peak to further electrochemical interrogations. Peak R1b which suffers the most interference from oxygen gives a poorer calibration profile. The lower limits of detection at peaks, R1a and R1b, were $2.7 \mu \mathrm{M}$ and $9 \mu \mathrm{M}$, respectively.

It can be seen from the calibration data presented here that the use of a CoPc-doped screenprinted sensor has significantly improved the magnitude of the current response when compared to the plain carbon sensors investigations [13], particularly at the lower levels of concentration, although this has not translated into greatly improved lower limits of detection.

\section{Cyclone collection of TNT}

We present here a short proof of principle study demonstrating the potential combination of electrochemical determination of TNT with a commercial cyclone system, to facilitate capture and subsequent measurement of air-borne traces of explosive materials. Prior to the collection of an explosive substance (TNT) the system was analysed for suitability using a well-defined sample redox probe, namely ferrocene carboxylic acid, and to assess the comparative concentrations of the analyte collected from the cyclone to that introduced into the system initially (i.e. the airborne concentration). The Coriolis ${ }^{\circledR} \mu$ collection system requires a collection solution to be present in the collection cone prior to sampling - this is to create the vortex into which any airborne particles will be collected through the centrifugal 
force exerted when the system is in activation. It was, therefore, necessary to add $10 \mathrm{ml}$ PBS into the collection cone prior to collection as the supporting electrolyte.

Solutions of FCA were interrogated using cyclic voltammetry at a CoPc electrode, as shown in figure S1, supplementary information. A calibration profile was obtained (figure S2). Similar solutions of varying concentrations were introduced into the cyclone collection system using a spray bottle to dispense samples as mist. The resulting sample was accumulated within the collection solution in the collection tube and interrogated electrochemically with an unmodified CoPc sensor. The voltammetric profile obtained is unmistakably due to FCA (figure 6), as the baseline PBS voltammetric profile shows no evidence of redox reaction. To assess the difference in the current response of the collected sample from that of an ideal sample (i.e. unaspirated), a study was performed using serial dilutions from a solution of $5 \mathrm{mM}$ FCA and interrogating them voltammetrically between 0.2 and $+0.65 \mathrm{~V}$ (vs. $\mathrm{Ag} / \mathrm{AgCl})$.

Several collections were conducted, introducing different concentrations of the FCA into the air sampling device using the spray bottle, and the collected sample solutions were subsequently interrogated electrochemically as described previously. The resulting voltammetric profiles (figure 7) demonstrate a correlation between observed electrochemical response with the different concentrations of the sample sprayed into the atmosphere in front of the air intake valve. It is obvious here that the current response obtained for solutions collected via the cyclone system are significantly lower than for a FCA sample tested from a stock solution (a $5 \mathrm{mM}$ stock solution gives a measured concentration of $0.028 \mathrm{mM}$ ).

The introduction of TNT into the air sampling system was performed as described for FCA using undiluted TNT solution, of $4.4 \mathrm{mM}$. This process was chosen both for its simplicity and also the fact that formulating a constant concentration of airborne TNT would be problematic. Also the intention of this experiment is to demonstrate the possibility of detecting "spikes" in TNT concentration, as would occur when an explosive device is passed through an area rather than the measurement of ambient levels. When TNT is collected with the air sampling system in the manner described previously and interrogated electrochemically, a distinct set of peaks are again visible (figure 7). The redox couple, known as R2 and O1, are clearly present as are the peaks in the cathodic region. Using the calibration curves, it is possible to calculate the collected concentration of TNT to be $390 \mu \mathrm{M}$. This corresponds to a recovery 
value of $101 \pm 6 \%$ (the error is due to the standard deviation of the electrochemical measurement), demonstrating a recovery that is statistically insignificant from optimum.

\section{Conclusions}

Visualisation of the topography of the plain carbon and cobalt phthalocyanine sensors was possible using SEM imaging and showed significant differences between the two surfaces, with the plain carbon sensors possessing a fairly featureless topography whilst the CoPc sensors showing flakes of what is postulated to be the cobalt phthalocyanine within the carbon ink matrix. Detection of TNT on CoPc sensors has been demonstrated here with a different current response profile when compared to plain carbon screen-printed sensors. The use of cobalt phthalocyanine sensors has improved response when using the main reductive peak R1a as a means for comparison, increasing the current response significantly. This peak is present in interrogations both with plain carbon and CoPc doped screen printed sensors, although the peak has become broader with more distinguishable features with the CoPc sensors. This may be due to the mediating effect of the cobalt phthalocyanine on the system.

When a PBS solution was interrogated electrochemically with the CoPc sensors there was a small reductive peak present at $-0.47 \mathrm{~V}$ (vs. $\mathrm{Ag} / \mathrm{AgCl}$ ) which was not apparent when interrogated with the plain carbon sensors. This peak is attributed to the electrochemical reduction of cobalt on the macrocyclic molecule. The effect of dissolved oxygen on the CoPc system is very much the same as observed when the nitroaromatic compounds were interrogated with the plain carbon sensors. Those reductive peaks in the far cathodic positions were observed to diminish with increasing deoxygenation periods whilst the redox couple at +0.01 and $-0.11 \mathrm{~V}$ were seen to increase.

This work has served to demonstrate the feasibility for integrating and coupling an air sampling device with an electrochemical detection system. Airborne FCA and TNT samples could be collected by the cyclone and transferred to an aqueous solution. The CoPc electrodes produced voltammetric profiles demonstrating the presence of TNT in the samples collected from the cyclone. While this collection technique coupled to the detection system developed over the course of this project has by no means been optimised, a proof of principle has been established here. Further work would be necessary to develop an optimised system. One challenge is that the vapour pressure for an explosive like TNT is low and so it 
may not be possible for an air sampling system to collect airborne particles of TNT concealed and well contained. However, explosives with a greater vapour pressure, such as triacetone peroxide, may be collected more easily. In the case of TNT, it would be necessary to improve the sensitivity of the detection system significantly to be able to detect airborne particles of a lower concentration than those demonstrated in this proof of principle piece. Selectivity of any electrochemical technique in unknown environmental samples is always an issue, however the high negative potential of the nitro group reduction means that very few organic species would interfere with this signal. We are currently investigating the use of modified surfaces to selectively bind and measure the species of interest - and this will form the basis of further publications on this study.

\section{Acknowledgement}

The authors would like to acknowledge the Engineering and Physical Sciences Research Council (EPSRC) for the funding and sponsorship of an Eng.D studentship for J.S.C. We would also like to thank Professor Paul Millner (Leeds University) for the loan of the cyclone.

\section{References}

[1] J. Wang, Electroanalysis 2007, 19, 415.

[2] J.S. Caygill, F. Davis, S.P.J. Higson, Talanta, 2012, 88, 14.

[3] K. Bratin, P.T. Kissinger, R.C. Briner, R. C. and C.S. Bruntlett, Anal. Chim. Acta, 1981, $130,295$.

[4] J. Wang, F. Lu, D. MacDonald, J. Lu, M.E.S. Ozsoz, K.R. Rogers, Talanta 1998, 6, 1405.

[5] L. Aguí, D. Vega-Montenegro, P. Yáñez-Sedeño and J.M. Pingarrón, Anal. Bioanal. Chem. 2005, 382, 381.

[6] K. Cizek, C. Prior, C. Thammakhet, M. Galik, K. Linker, R. Tsui, A. Cagan, J. Wake, J.L. Belle, J. Wang, Anal. Chim. Acta, 2010, 661, 117.

[7] J. Chen, J. Shih, J.C. Liu, M. Kuo, J. Zen, Anal. Chem. 2006, 78, 3752. 
[8] M. Chuang, J.R. Windmiller, P. Santhosh, G.V. Ramírez, M. Galik, T. Chou, J. Wang, Electroanalysis. 2010, 22, 2511.

[9] J. Wang, M. Pumera, Talanta 2006, 69, 984.

[10] S. Hrapovic, E. Majid, Y. Liu, K. Male, J.H.T. Luong, Anal. Chem. 2006, 78, 5504.

[11] M.S. Goh, M. Pumera, M. Anal. Bioanal. Chem. 2011, 399, 127.

[12] S. Guo, D. Wen, Y. Zhai, S. Dong, E. Wang, Biosens. Bioelec. 2011, 26, 3475.

[13] J.S. Caygill, S.D. Collyer, J.L. Holmes, F. Davis, S.P.J. Higson, Analyst, 2013, 138, 346.

[14] J.P. Whelan, A.W. Kusterbeck, G.A. Wemhoff, R. Bredehorst, F.S. Ligler, Anal. Chem. 1993, 65,3561 .

[15] S. Maree, T. Nyokong, J. Electroanal. Chem, 2000, 492, 120.

[16] J.P. Hart, I.C. Hartley, Analyst, 1994, 119, 259.

[17] N. Kobayashi, P. Janda, A.B.P. Lever, Inorg. Chem. 1992, 31, 5172.

[18] F. Wang, W. Wang, B. Liu, Z. Wang, Z. Zhang, Talanta, 2009, 79, 376.

[19] K.C. Honeychurch, J.P. Hart, P.R.J. Pritchard, S.J. Hawkins, N.M. Ratcliffe, Biosens. Bioelec. 2003, 19, 305. 
List of figures.

Figure 1. An SFEG scanning electron micrograph of a). a bare carbon and b). a cobalt phthalocyanine doped screen-printed electrode surface at 2000x magnification (after sputter coating with gold palladium). (c) Four-electron reduction of nitro groups on TNT to hydroxylamine and further two-electron reduction from hydroxylamine to amine

Figure 2. Cyclic voltammogram of $200 \mu \mathrm{M}$ TNT on a plain carbon and a CoPc doped sensor platform as well as (inset) of $\mathrm{pH}$ 7.0 PBS solution on a CoPc doped and plain screen printed carbon sensor, (at a scan rate of $20 \mathrm{mVs}^{-1}$ vs. $\mathrm{Ag} / \mathrm{AgCl}$ without deoxygenation).

Figure 3. Cyclic voltammograms for varying concentrations of TNT on a CoPc doped screenprinted carbon (in PBS at a scan rate of $20 \mathrm{mV} \mathrm{s}^{-1}$ ).

Figure 4. Voltammograms demonstrating the influence of increasing deoxygenation periods on a $100 \mu \mathrm{M}$ TNT solution in a PBS solution (at a scan rate of $20 \mathrm{mVs}^{-1} \mathrm{vs}$. $\mathrm{Ag} / \mathrm{AgCl}$ ).

Figure 5. Calibration plots for TNT in PBS solution on a screen-printed carbon CoPc electrode taken from the peak in position at $+0.01 \mathrm{~V},-0.11 \mathrm{~V},-0.50 \mathrm{~V}$ and $-0.77 \mathrm{~V}$ (vs. $\mathrm{Ag} / \mathrm{AgCl})-$ standard deviations $(\mathrm{n}=3)$ are shown.

Figure 6. Voltammograms obtained from the electrochemical interrogation of FCA in a PBS solution after aspiration and collection using the air sampling system, with a CoPc sensor (scan rate $20 \mathrm{mVs}^{-1}$ vs. $\mathrm{Ag} / \mathrm{AgCl}$ )

Figure 7. Voltammograms obtained from the electrochemical interrogation of $4.4 \mathrm{mM}$ TNT after aspiration and collection using the air sampling system, with a CoPc sensor (scan rate 20 $\mathrm{mV} \mathrm{s}^{-1}$ vs. $\left.\mathrm{Ag} / \mathrm{AgCl}\right)$.

Table 1. Data from $200 \mu \mathrm{M}$ TNT interrogated with a) a SPCE and b) comparison data of nitroaromatic compounds interrogated with a cobalt phthalocyanine doped screen-printed electrode. Peak potential $\left(E_{i}\right)$ is in volts and peak current $\left(i_{p}\right)$ is in $\mu A$. 
Table 1.

a)

\begin{tabular}{ccccccc}
\hline SPCE & O1 $\mathbf{E}_{\mathbf{i a}}$ & $\mathbf{O 1} \mathbf{i}_{\mathbf{p a}}$ & $\mathbf{R 2} \mathbf{E}_{\mathbf{i c}}$ & $\mathbf{R 2} \mathbf{i}_{\mathbf{p c}}$ & $\mathbf{R} 1 \mathbf{E}_{\mathbf{i c}}$ & $\mathbf{R} 1 \mathbf{i}_{\mathbf{p c}}$ \\
\hline TNT & +0.3 & 0.89 & -0.3 & 0.53 & -0.72 & 17.16 \\
\hline
\end{tabular}

b)

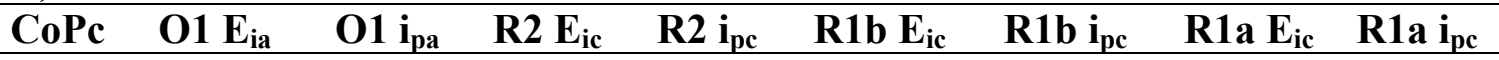

\begin{tabular}{lllllllll} 
TNT & +0.01 & 13.50 & -0.11 & 7.77 & -0.5 & 33.79 & -0.77 & 50.96 \\
\hline
\end{tabular}

Table 1. Data from $200 \mu \mathrm{M}$ TNT interrogated with a) a SPCE and b) a cobalt phthalocyanine doped screen-printed electrode. Peak potential $\left(E_{i}\right)$ is in volts and peak current $\left(i_{p}\right)$ is in $\mu \mathrm{A}$. 
Figure 1.
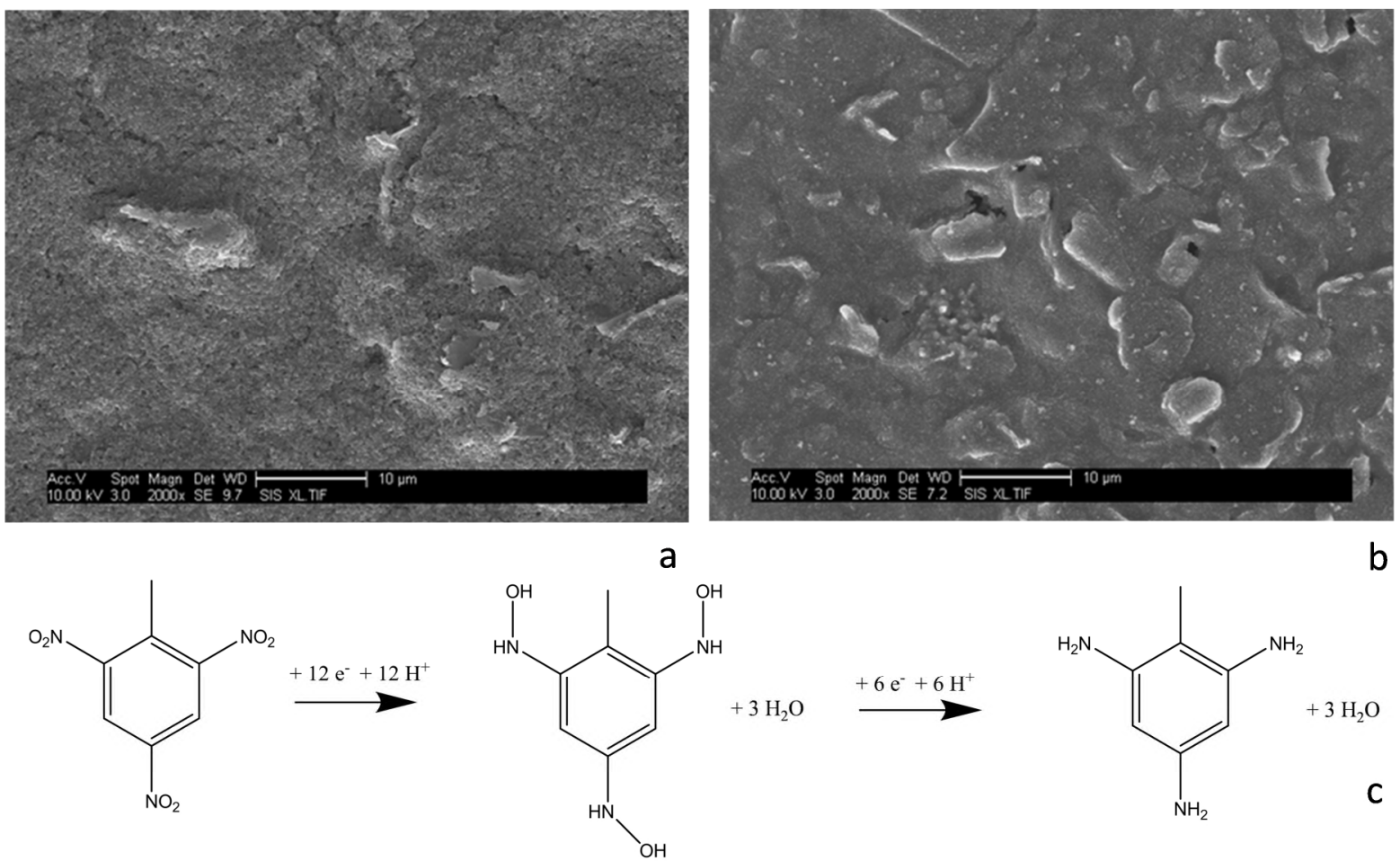
Figure 2.

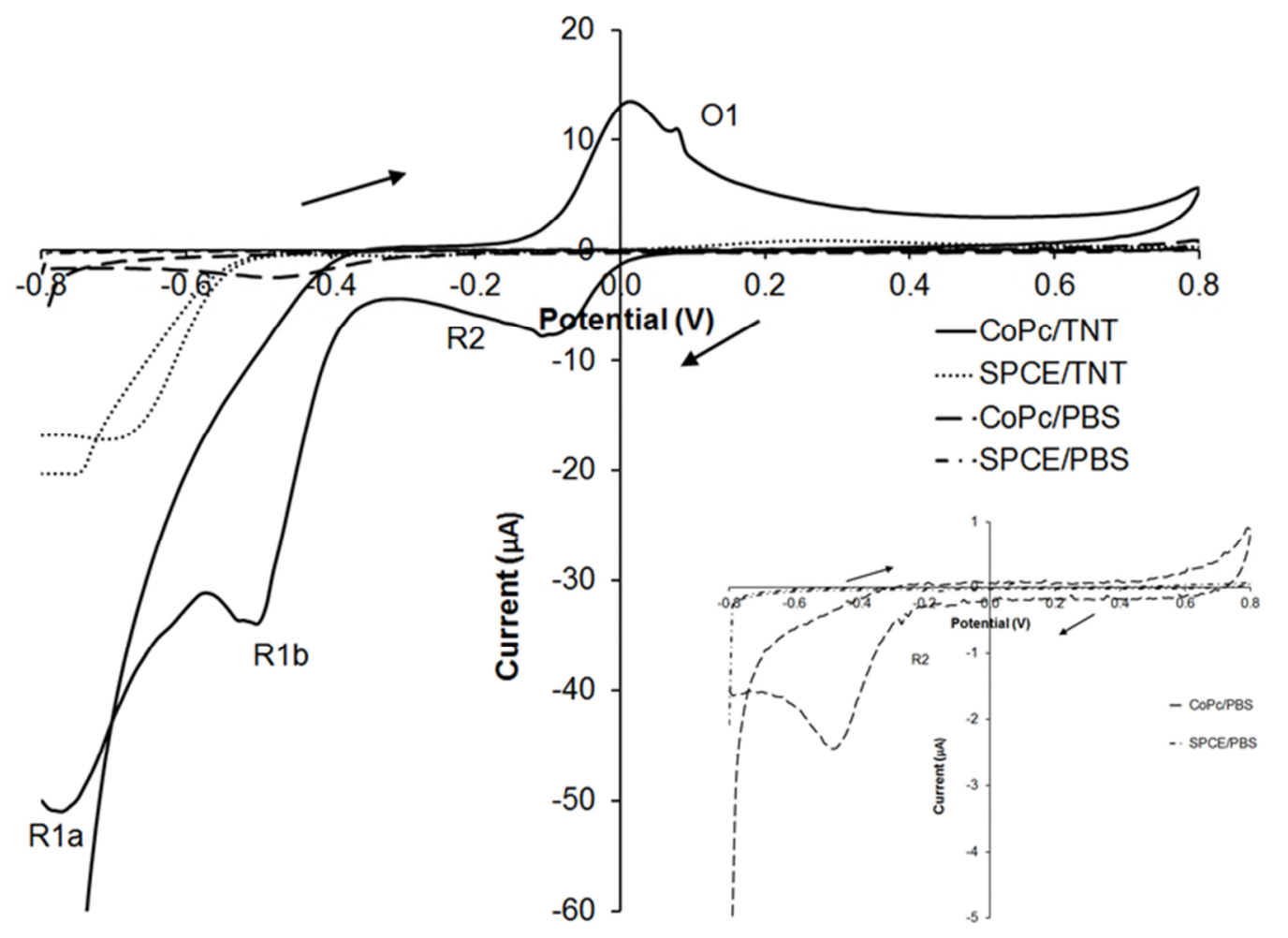


Figure 3.

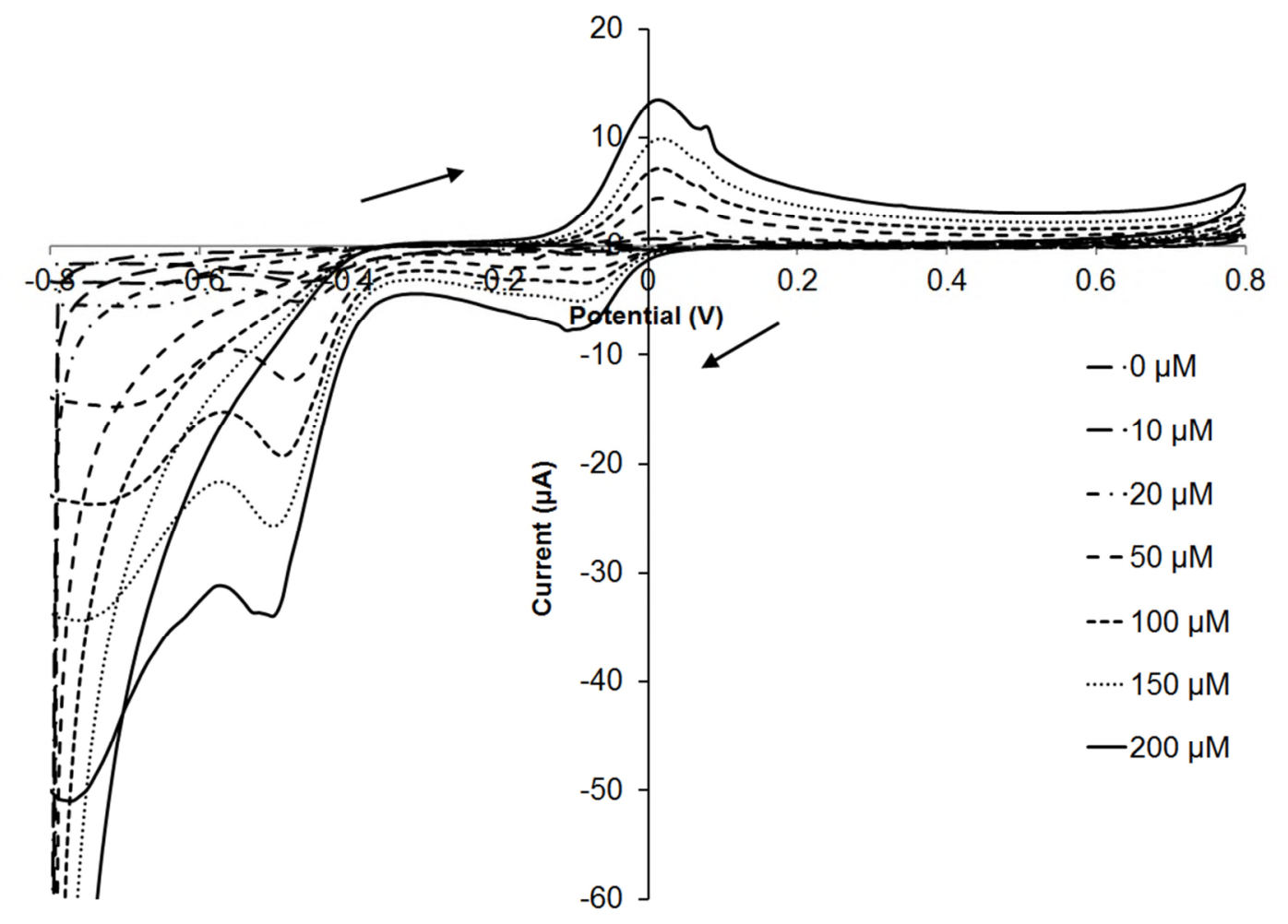


Figure 4.

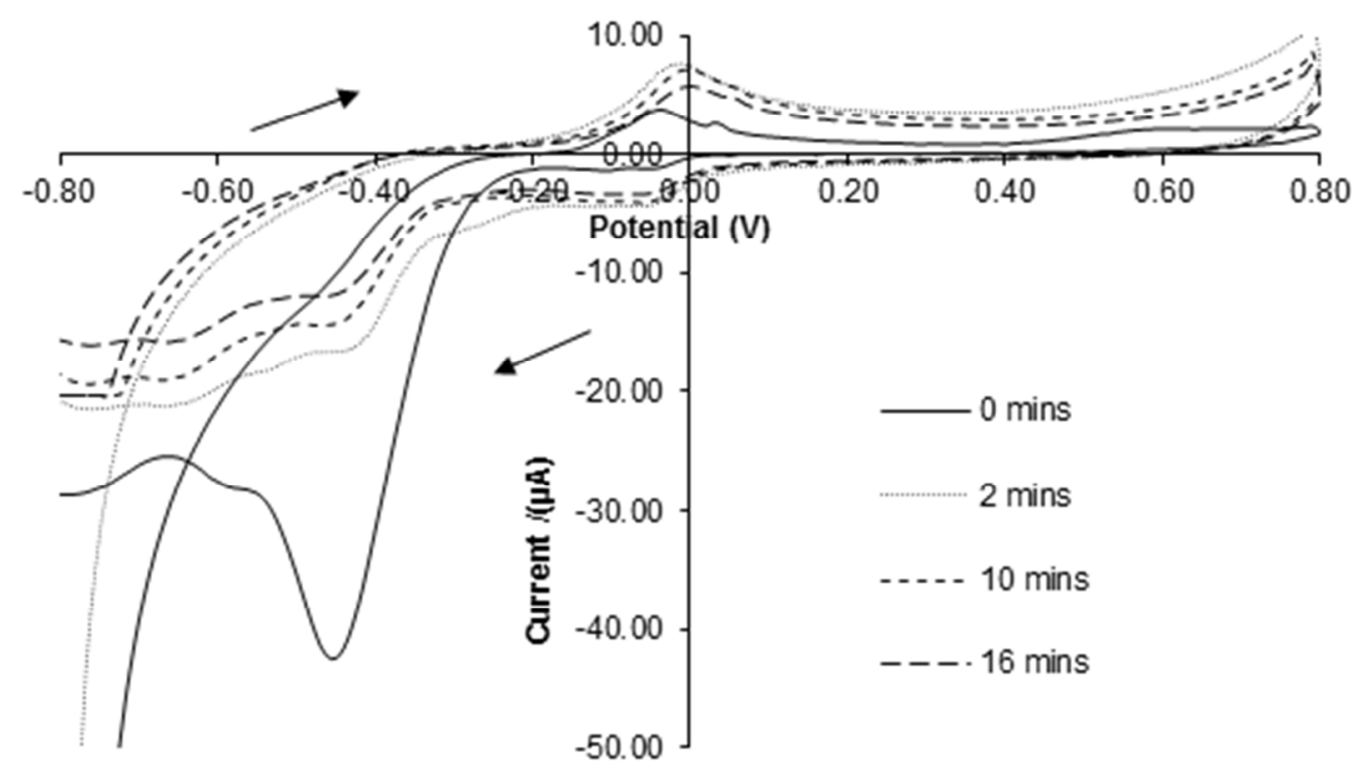


Figure 5.
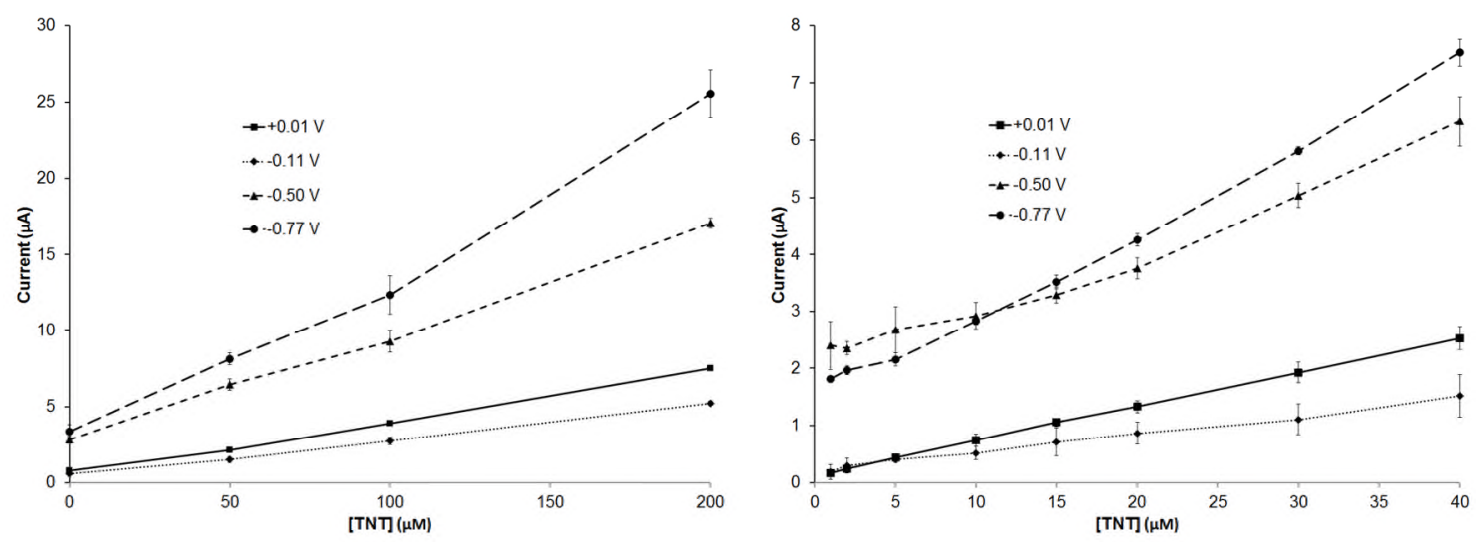
Figure 6.

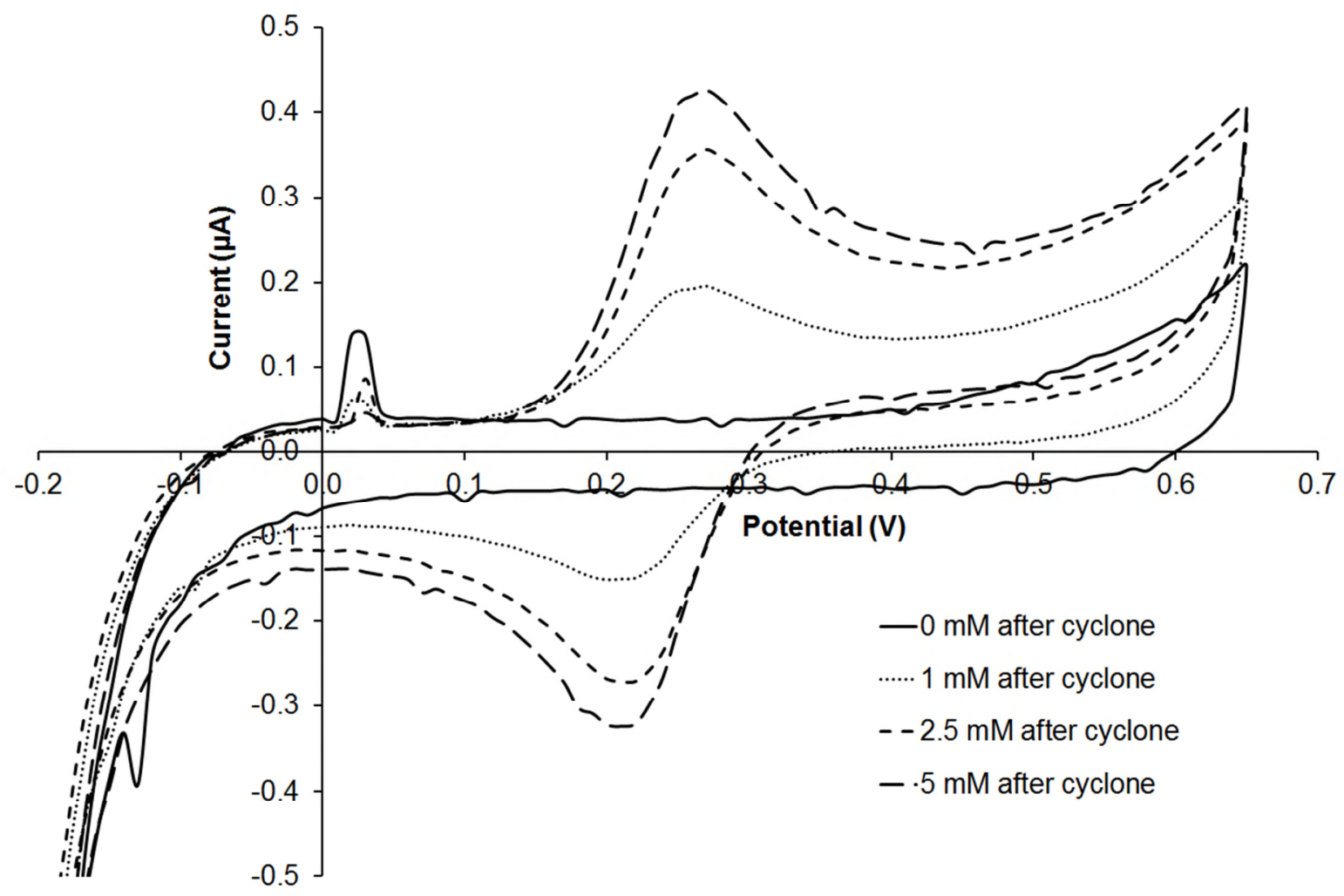


Figure 7.

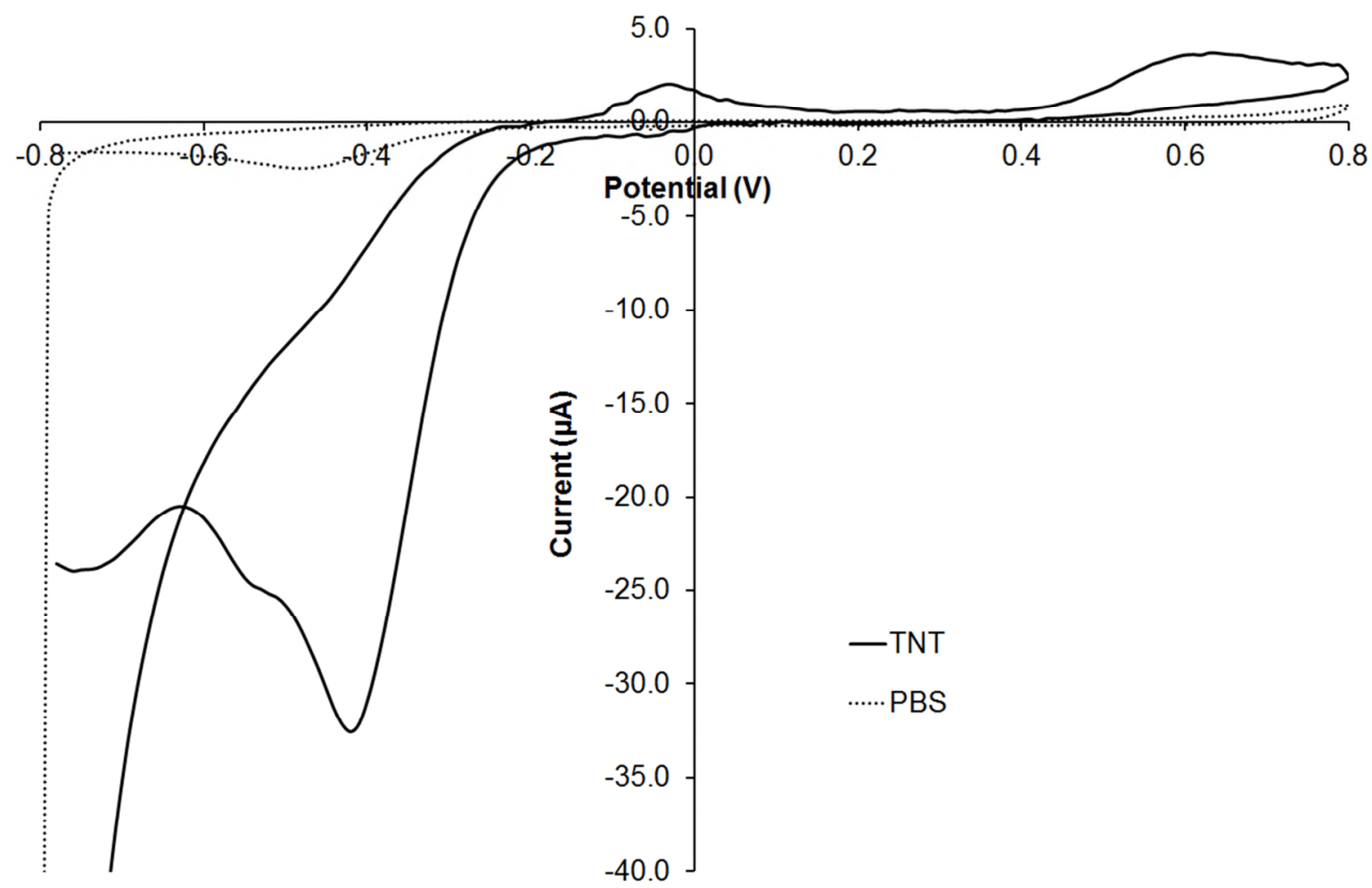


Disposable screen-printed sensors for the electrochemical detection of TNT and DNT.

J. Sarah Caygill ${ }^{1}$, Stuart D. Collyer ${ }^{2}$, Joanne L. Holmes ${ }^{1}$, Frank Davis ${ }^{1}$, Séamus P.J. Higson ${ }^{1 *}$ ${ }^{1}$ Cranfield Health, Vincent Building, Cranfield University, Bedfordshire, MK43 OAL, UK.

${ }^{2}$ Microarray Ltd, PO Box 88, Manchester, M60 1QD, UK.

* Corresponding author. Tel: +44(0)-1234-758516. Fax: +44(0)-1234-758380. Email address: s.p.j.higson@cranfield.ac.uk

\section{Supplementary information}

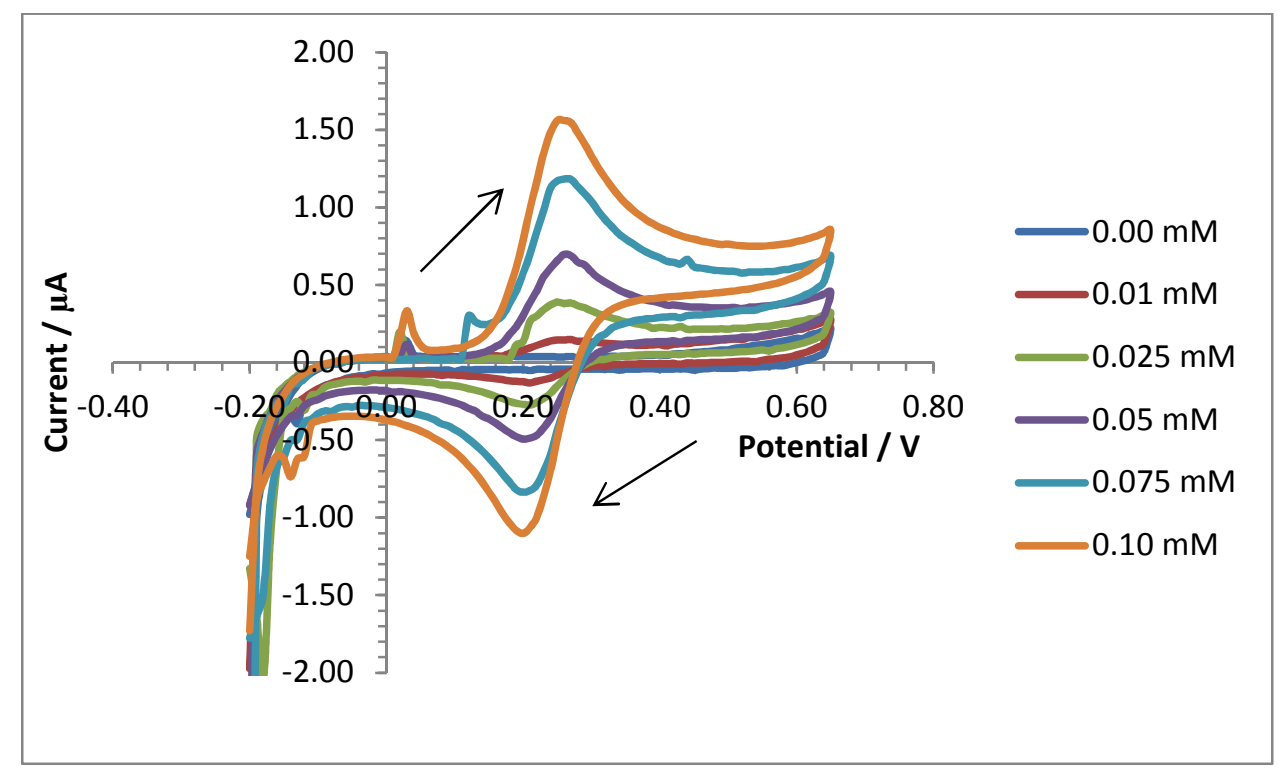

Figure S1. Voltammograms obtained from the electrochemical interrogation of a variety of concentrations of ferrocenemonocarboxylic acid in a phosphate buffer solution, with a CoPc sensor (scan rate $20 \mathrm{mVs}^{-1}$ vs. $\mathrm{Ag} / \mathrm{AgCl}$ ) 


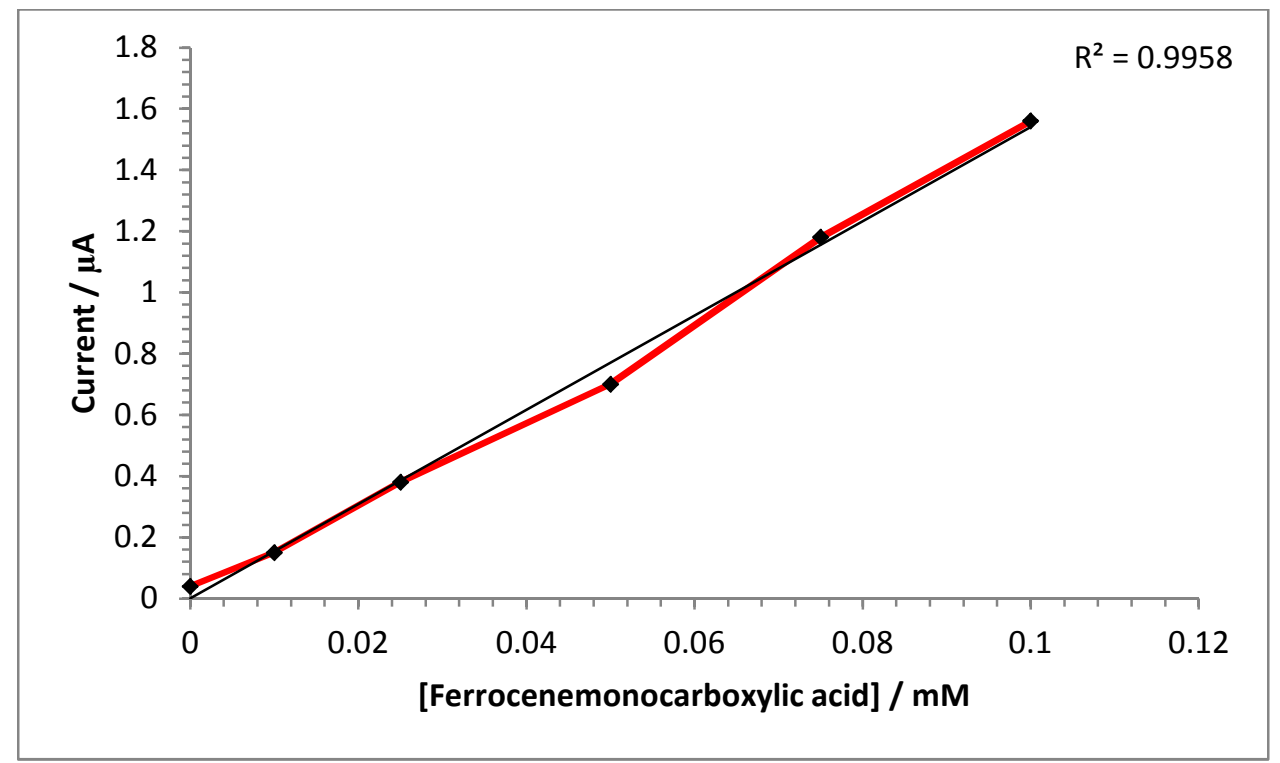

Figure S2 Calibration plot taken from the maximum anodic peak current of a variety of concentrations of pure ferrocenemonocarboxylic acid in a phosphate buffer solution (scan rate $20 \mathrm{mVs}^{-1}$ vs. $\left.\mathrm{Ag} / \mathrm{AgCl}\right)$ 
2013-10-11

\section{Electrochemical detection of TNT at cobalt phthalocyanine mediated screen-printed electrodes and application to detection of airborne vapours}

Caygill, J. S.

Wiley-Blackwell

Caygill, J. S. et. al (2013) Electrochemical detection of TNT at cobalt phthalocyanine mediated screen-printed electrodes and application to detection of airborne vapours, Electroanalysis, Vol. 25, Iss. 11 , pp. $2445-2452$

10.1002/elan.201300327

Downloaded from Cranfield Library Services E-Repository 